\title{
Modifiye cam iyonomer simanlar: Güncel bir yaklaşım
}

\author{
Mustafa Erhan Sarı(0000-0002-7837-1069) ${ }^{\alpha}$, Sevgin İbiş(0000-0002-2800-2214)
}

Selcuk Dent J, 2019; 6: 206-212 (Doi: 10.15311/selcukdentj.310844)

Bašuru Tarihi: 06 Mayıs 2017 Yayına Kabul Tarihi: 31 Mayıs 2018

\begin{abstract}
öz
Modifiye cam iyonomer simanlar: Güncel bir yaklaşım

Cam iyonomer simanların biyolojik uyumlulukları ve flor salma özellikleri ile diş hekimliğinde pek çok uygulama endikasyonu bulunur. Son yillarda bu materyallerin fiziksel ve kimyasal özelliklerinin geliștirilebilmesi için, rezin modifiye cam iyonomerlere nano-boyutlu doldurucuların eklenmesi, cam partiküllerinin boyutunun azaltıması ve cam tozuna nano-boyutlu biyoseramiklerin sokulması gibi çeşitli yenilikler tanıtılmıştır. Bu yenilikler geleneksel cam iyonomerlerin mekanik özelliklerini, aynı zamanda flor salınımını ve biyoaktivitesini de arttırmaktadır. Bu derlemenin amacı bu yenilikleri gözden geçirmektir.
\end{abstract}

\section{ANAHTAR KELIMELER}

Adeziv diş hekimliği, cam iyonomer siman, nano teknoloji

Modern dental materyallerin iyi bir örneği olan cam iyonomer simanlar (CIS), 1972 yilinda ASPA (aluminosilicatepolyacrylic asit) adı altında Wilson ve Kent $^{1}$ tarafından tanıtımıştır. Silikat simanla, polikarboksilat simanın hibriti şeklinde tanımlanan cam iyonomer simanlar toz-likit formların karıştıııması ile elde edilir. Toz formu, bazik floro-alumino silikat taneciklerinden, kalsiyum, florür, sodyum ve fosfattan oluşurken; likiti ise aköz poliakrilik asitten oluşur. ${ }^{2}$

CIS'ların karakteristik özelliklerinden biri asit polimer zincirlerinin karboksil gruplarının mine ve dentindeki kalsiyum iyonları ile şelasyonu vasıtasıyla gerçekleşen diş dokularına kimyasal bağlantısıdır. ${ }^{3}$ Buna ek olarak kabul edilebilir translüsentliğe sahip olması, flor iyonu salabilmeleriyle bağlantılı çürük karşıtı etkilerinin olması, diş dokularıyla termal uyumluluk göstermesi, herhangi bir bondlama basamağı olmadan yerleştirilebilmesi ve biyouyumlu olması gibi avantajları olan bu materyaller diş hekimliğinde geniş bir uygulama alanına sahiptir. ${ }^{4,5}$

Süt dişlerinin daimi restorasyonlarında, sınıf 3 ve sınıf 5 restorasyonlarda, daimi molarlarda fissür örtücü olarak, kron, köprü, yer tutucu ve ortodontik malzeme yapıştırıması ve diğer indirekt restorasyonların yapıştırımasında, sandviç tekniği ve atravmatik restoratif tedavi materyali olarak kullanılmaktadır. ${ }^{2,6-11}$ Bütün bu

\section{ABSTRACT}

Modified glass ionomer cements: A current approach

As their biological compatibility and fluoride ion release, glass ionomer cements are being used in several clinical applications in dentistry. In recent years, various innovations have been introduced to improve the physical and chemical properties of these materials such as incorporation of nano-sized fillers to resin modified glass ionomer cement, reducing the size of the glass particles, and introducing nano-sized bioceramics to the glass powder. These innovations increase the mechanical properties of conventional glass ionomers, as well as fluoride release and bioactivity. The aim of this paper is to review these modifications.

\section{KEYWORDS}

Adhesive dentistry, glass ionomer cement, nanotechnology

avantajlarının yanında aşınma direncinin düşük olması, nem kontaminasyonuna hassas olması, yüksek oranda mikro sızıntı göstermesi ve yüzey özelliklerinin yetersiz olması gibi dezavantajları olan bu materyallerin çalışma zamanı kısa ve sertleşme zamanı uzundur. ${ }^{12}$

$\mathrm{Bu}$ derlemenin amacı CIS'ların zayıf mekanik ve fiziksel özelliklerini geliştirebilmek için yapılan çeşitli modifikasyonları gözden geçirmektir.

\section{Toz-modifiye nano cam iyonomerler}

Nano-teknoloji 1-100 nm aralığındaki sistemlerin, modifikasyonların ve materyallerin kullanımını içermektedir. ${ }^{13-15}$ İmplant yüzey modifikasyonları, nano-size partiküllerin katılığı desteklenmiş polimerik kompozitlerin üretimi diş hekimliğindeki nano teknoloji uygulamalarına örnek olarak gösterilebilir. ${ }^{15-17}$ Son zamanlarda nano-teknoloji kullanılarak nano-boyutlu parçacıklar veya nanokümelerin, rezin kompozitlere eklendiği gibi ${ }^{18-20}$ mekanik ve fiziksel özelliklerini geliştirerek CIS'lara eklenmesi de denenmiştir ${ }^{21,22}$

Nano-boyutlu partiküllerin üretilmesinde yukarıdan aşağıya ve aşağıdan yukarıya olmak üzere iki

\footnotetext{
${ }^{\alpha}$ Ondokuz Mayıs Üniversitesi, Diş Hekimliği Fakültesi, Pedodonti Anabilim Dalı, Samsun

$\beta$ Bafra Ağız ve Diş Sağlığı Merkezi, Çocuk Diş Hekimliği Kliniği, Samsun
} 
yaklaşım vardır. ${ }^{14}$ Yukarıdan aşağıya nanofabrikasyon; öğütme, aşındırma ve taş baskı gibi kütle materyallerin kaldırıarak, nano-boyutlu partiküllerin üretimini içerir. Aşağıdan yukarıya nano-fabrikasyon ise, nano-boyutlu partikül atomu üretmeyi içerir ve bu nano-fabrikasyona örnekler doku rejenerasyonu, protein sentezi ve biyomimetik dental implant kaplamalarıdır. CIS'lara katılmak üzere nano-boyutlu partikül üretimi, bulk materyallerin apatit, silikat cam ve bazı metal oksitlerin yukarıdan aşağıya nanofabrikasyonu ile gerçekleștirilir. ${ }^{23}$ De Caluwe ve ark. ${ }^{24}$ çalışmalarında konvansiyonel cam iyonomerlere nano-boyutlu cam partiküllerin eklenmesinin hazırlama süresini azalttığı, sıkışma direncini ve elastisite modülünü arttırdığını göstermişlerdir.

Ağız ısısının ve nemin etkisini belirlemek için laboratuvar ortamında, termal siklus gibi yöntemlerle restoratif materyallerin yapay yaşlanmaları sağlanmıştır. Termal siklus; nano dolduruculu CIS'ların mekanik özelliklerine konvansiyonel CIS'lara göre daha fazla zararlı etki göstermiştir ve bu durum bu tür materyallerin uzun vadeli ömrünü olumsuz etkileyebileceği fikrini oluşturmuştur. Cam iyonomerlerin kimyasal yapıları spektroskopik yöntemlerle de test edilebilir. Apatit nano kristallerin katılmasıyla olan nano modifikasyonun CIS'lara etkisi 'Fourier Transform Spektroskopi (FTIR)' ile gözlemlenebilirken ${ }^{21,25}, \quad \mathrm{TiO}_{2}$ ve $\mathrm{ZrO}_{2}$ gibi diğer partiküllerin kullanılmasıyla yapılan modifikasyonların etkisi henüz araştırılmamış veya rapor edilmemiştir.

\section{Nano apatit modifikasyonu}

Hidroksiapatit ve floroapatit kristalleri mineralize kemik ve dental dokuların benzer yapıları nedeniyle, çürük önlemede remineralizasyona destek olarak ve implant teknolojisi gibi diş hekimliğinin birçok alanında kullanılmaktadır. ${ }^{15,26-29}$ Son zamanlarda nanohidroksiapatit ( $\mathrm{nHAp}$ ) eklenerek modifiye edilmiş olan rezin kompozitlerin, modifiye edilmemiş rezin kompozitlere göre daha üstün mekanik özelliklere sahip olduğu gözlemlenmiştir. ${ }^{30,31}$ Benzer şekilde konvansiyonel CIS'ların tozuna nanohibrit veya nanofloroapatit (nFAp) eklenmesi; basma, çekme ve eğilme kuvvetlerine karşı materyalin mekanik özelliklerini olumlu yönde etkilemiştir. ${ }^{25}$

CIS tozuna apatit eklenmesinin hazırlanma sırasında kristalliği arttırdığı FTIR spektroskopisi ile ortaya konulmuştur ve bu sayede kimyasal stabilite ve suda çözünürlük iyileşmiştir. nFAp, nHAp'den daha düşük çözünürlük gösterdiği için nFAp içeren CIS'lar, nHAp içeren CIS'lardan daha iyi mekanik özelliğe sahiptir. ${ }^{21,25}$ Apatit modifiye CIS'ların gelişmiş mekanik özelliklerinin, poliakrilik asit ve apatit kristalleri arasındaki iyonik etkileşim sonucu olduğu ileri sürülmüştür. ${ }^{21}$
Standart poliakrilik kopolimerlerin yerine, nHAp içeren toza $n$-vinilpirilidon, itatonik asit ve poliakrilik asit eklendiğinde iyileştirme daha belirgindir. $\mathrm{Bu}$ da $\mathrm{n}$ vinilpirilidon ve apatit kristalleri arasındaki fiziko-kimyasal etkileşime bağlanmışır. ${ }^{25}$ Dahası, diş dokularındaki kalsiyum iyonları ile apatit kristalleri arasındaki güçlü iyonik bağ oluşumuna bağlı olarak nano-apatit içeren cam iyonomerler, diş yüzeyine daha yüksek bağlantı göstermektedir. ${ }^{32}$ Buna ek olarak apatit boyutlarının azalması yüzey alanını arttırır ve kristallerin demineralize dentine ve mine boşluklarına infiltrasyonu artar. Böylece diş-iyonomer ara yüzü bağlantısı gelişmiş olur. ${ }^{33}$

Nano-boyutlu $\mathrm{HAp} / \mathrm{Zr}, \mathrm{CaF}_{2}$ ve $\mathrm{TiO}_{2}$ partikülleri ile modifikasyon

CIS'ların mekanik özelliklerini geliştirmek için tozuna \% 4 konsantrasyonda zirkonya ve $\mathrm{HAp}\left(\mathrm{HAp} / \mathrm{ZrO}_{2}\right)$ kombinasyonu eklenmiştir ve CIS örnekleri SEM ile analiz edilmiştir. Matriks içinde fark edilen yoğun ve homojen dağılım gösteren cam ve $\mathrm{HAp} / \mathrm{ZrO}_{2}$ partikülleri, mekanik özelliklerin geliştirilmesi için önemli bir faktör olmuştur. Bununla birlikte zayıf $\mathrm{ZrO}_{2}$-cam ara yüzeyi bağlantısına bağlı olarak modifiye edilmemiş CIS'lara göre daha fazla çatlak görülmüştür. Zayıf $\mathrm{ZrO}_{2}$-cam ara yüz bağlantısına ve daha az cam oranına bağlı olarak, \% 4'ü aşan $\mathrm{HAp} / \mathrm{ZrO}_{2}$ konsantrasyonu, CIS'ların özelliklerine olumsuz etkide bulunmaktadır. ${ }^{34}$ Kalsiyum florid $\left(\mathrm{CaF}_{2}\right)$ nanopartikülleri de RMCIS'lara katılarak mekanik özellikler geliştirilebilir ve CIS'ların flor salınım yeteneği üzerine olumlu etkide bulunabilmektedir. ${ }^{35}$ Benzer şekilde CIS tozuna $\mathrm{TiO}_{2}$ (\% 3-5 wt.) nanopartikülleri eklenmesinin materyalin antibakteriyel etkisini ve mekanik özelliklerini artırdığı gözlemlenmiştir. ${ }^{36,37}$ CIS'ların toksisitesini değerlendirmek için in vitro olarak yapılan çalışmalarda nano-partiküllü $\mathrm{TiO}_{2}$ içeren CIS'ların prostaglandin $E_{2}$ inflamatuar faktörünün salınımını stimüle ettiği görülmüştür. ${ }^{38}$ Sitotoksik etkisinden dolayı bu materyallerle ilgili daha geniş çalışmaların yapılması önerilmektedir.

\section{Nano dolduruculu rezin modifiye cam iyonomerler}

Cam tozu ve poliasit solüsyondan meydana gelen konvansiyonel CIS'ların aksine, RMCIS'lar kendi kendine veya ışıkla aktive olan polimer rezin komponentler içerir. Bu hibrit materyaller, CIS'ların çürük önleyici potansiyeli ile mekanik özelliklerini birleştirmek için geliştirilmiştir. ${ }^{12}$ Aslında RMCIS'ların flor salma özelliklerinin yanında aynı zamanda konvansiyonel CIS'lara göre üstün bükülme dirençleri vardır ve bu materyaller daha az çözünürlüğe sahiptir. RMCIS'ların yerleştirilmesinden sonraki ilk 24 saat su emilimine bağlı genişlemelerinin \% 3.4'den \% 11.3'e çıktığı görülür. ${ }^{39}$ Rezin kompozitlerle karşılaştııılığında konvansiyonel RMCIS'lar, daha kırılgandır ve daha az estetiklerdir. ${ }^{12,39}$ Buna ek olarak konvansiyonel toz bazlı cam iyonomerlerle karşıllaştııılığında RMCIS'lar azalmış flor salınımına sahiptir ancak daha yüksek yayılma gösterirler. ${ }^{40}$ 
Nano-RMCIS'ların mekanik, fiziksel özellikleri ve flor salınımı

Kompozit restoratif materyallere benzer şekilde CIS'larda da doldurucu partiküllerin boyutu ve şekli mekanik özelliklerini etkiler. ${ }^{34,41}$ Genel olarak doldurucuların daha düşük boyutlu ve daha yoğun olması CıS'ların sıkışma dayanımını ve sertliğini arttııı ayrıca partiküllerin büyümesi daha yüksek aşınma direnci sağlar. ${ }^{41} \mathrm{RMCIS}$ 'lardaki cam partiküller ve rezin faz arasındaki kimyasal bağlantı, konvansiyonel CIS'lara göre daha yüksek bükülme ve çekme direncine sahip olmalarını sağlar. ${ }^{41,42}$

Konvansiyonel RMCIS'lar, nano-RMCIS'lara göre nispeten daha iyi bükülme dayanımı ve daha yüksek yorulma sınırı gösterirler. ${ }^{35,43}$ Buna ek olarak nanoRMCIS'lar asitle pürüzlendirildiğinde mekanik testlerde daha kötü performans göstermişlerdir. Çünkü asidojenik mikrofloraya bağlı olarak oral $\mathrm{pH}$ 4'e kadar düşebilir ve asidik ortam bu malzemelerin asit erozyonuna duyarlılığı nedeniyle nano-RMCIS'ların uzun vadeli ömrünü tehlikeye sokabilir. ${ }^{44}$ Buna ek olarak rezin polimerizasyonu sırasındaki nanopartiküller arasındaki boşluklardan kaynaklı olarak nHAp modifiye RMCIS'ların hazırlama süresi 800 saniyeyi aşabilir ve bu süre ISO standartlarından (90-480s) daha uzundur. ${ }^{45,46} \mathrm{RMCIS}$ 'lar dentine \% 10'luk poliakrilik asit kullanılarak açılan kollajen bağlarının içine "mikromekanik bağlar" ve demineralize dentin/minenin içindeki HAp kristallerinin, kalsiyum iyonlarıly asitten gelen karboksil gruplarının iyonik etkileşimiyle meydana gelen "kimyasal bağlanmanın" kombinasyonuyla bağlanır. ${ }^{47,48}$ Nano dolduruculu RMCIS'lar da benzer bir bağlanma mekanizması gösterir ancak dentine rezin tagların minimal infiltrasyonu, konvansiyonel CIS'lara benzer şekilde mikromekanik tutunmadan ziyade dişlerle olan iyonik bağlantının göstergesidir. ${ }^{49}$

Ticari olarak temin edilebilen nano-dolduruculu RMCIS (Ketac N100/Ketac Nano 3M ESPE, St. Paul, MN, USA) silika doldurucu nanokümeleri içerir ve bir primer (Ketac Nano Primer) ile tedarik edilir. Cam iyonomer simanın yerleştirilmesinden önce diş dokularına bu primer uygulanır. Diğer yandan son çalışmalar göstermiştir ki nano-dolduruculu RMCIS ve konvansiyonel RMCIS'lar arasında çekme dayanımı açısından anlamlı fark bulunamamıştır. ${ }^{49}$ Genel olarak bilindiği üzere aşındırma prosedürlerini takiben dentinin aşırı kurutulması kollajen fibrillerin çökmesine neden olabilir. Wet-bonding teknik dentin yüzeyinin nemli kalmasını sağlar ve bu durum havayla kurutmanın ortaya çıkardığı demineralize dentinin çökmesini engeller. Bununla birlikte fonksiyonel primerlerle spesifik dentin hazırlığı da; dentin kollajen fibrillerinin çökmesini önler ve RMCIS'lar gibi rezin adezivlerin bağlanma performansını arttırır. ${ }^{50,51}$
Diş preparasyonunu takiben diş yüzeyinde kalan bir tür film olarak tanımlanan smear tabakası, restoratif materyallerin diş dokularına bağlanmasına engel olmaktadır. ${ }^{52}$ Diğer yandan fonksiyonel primerlerin pH'ı smear tabakasını kaldırmak için yeteri kadar düşüktür ve dentin ve mineye kimyasal bağlanmayı arttırabilir. ${ }^{53,54}$ Benzer şekilde \% $37^{\prime}$ lik fosforik asitle pürüzlendirme; smear tabakasını kaldırarak ve yüzey enerjisini arttrarak dolduruculu RMCIS'ların makaslama direncini yükseltebilir. ${ }^{54-56}$

Bununla birlikte, GIC'lerin ve RMGIC'lerin; fosforik asitle dekalsifiye dentinin infiltrasyonundan hariç tutulan nispeten yüksek moleküler ağırlıklı polikarboksilik asit bazlı polimerler (Mw: 8000 ila $15,000)$ içerdiği göz önünde bulundurulmalıdır. ${ }^{57,58}$ Buna göre dekalsifye dentinde kollajen ağlar korunmasız kalabilir ve hidrolitik bozulmaya maruz kalabilir. CIS bazlı materyallerin polialkenoik polimerlerinin dentin kollajeninin içine nüfuz edememesi sebebiyle agresif dentin ön işlemleri bu materyaller kullanilırken uygulanmamalıdır. ${ }^{59} \mathrm{Bu}$ nedenle nano-dolduruculu RMCIS'ların restorasyonları sırasında, ek bondlama basamağı veya poliakrilik asit ajan uygulanmasının bu materyallerin yapışma performansını arttırdığına inanılmaktadır. Lazerle asitleme de bu nanodolduruculu RMCIS'lar için potansiyonel bir bağlama yöntemi olarak gösterilse de geleneksel yöntemlerle karşılaştıııldığında bağlanma dayanımı açısından anlamlı bir gelişme gözlenmemiştir. ${ }^{60}$ NanoRMCIS'ların klinik performanslarını konvansiyonel CIS'lar ve rezin kompozitlerle karşılaştıran bir çalışmada restorasyonlar arasında bir yıllık sağ kalım açısından fark olmadığı gösterilmiştir. ${ }^{61}$ Bununla birlikte, nano-RMGIC'nin konvansiyonel RMGIC'ye kıyasla daha düşük marjinal bütünlüğe sahip olduğu bulunmuştur ve bu düşük bağlanma performansı nano-RMCIS'ların dentin yerine mineye uygulanmasına bağlanmıştır. ${ }^{62}$

Yüksek konsantrasyonlarda florun demineralizasyonu azaltıp remineralizasyonu artırdığı, diş yüzeyine bakteri gelişimini ve yapışmasını engellediği ayrıca kompleks bakteri biyofilminin oluşmasını durdurduğu bilinmektedir. ${ }^{5}$ Cam iyonomerlerden flor salınımı bilinen bir durumdur. Ancak iyonomerler içindeki flor, sertleşme reaksiyonunda rol almaz, iyon değişimi süresinde salınır. Dahası cam iyonomerler tükürükteki floru absorbe edebilir ve flor rezervi olarak görev alabilir. ${ }^{40,63-67}$ Ancak bugüne kadar cam iyonomerlerden salınan flor miktarının çürük oluşumunu engellemek için yeterli olduğu kanitlanamamıştır. ${ }^{66,67}$ Nano-RMCIS ve konvansiyonel RMCIS'lardan salınan kütlesel floridleri birbirleriyle karşıllaştıran çeşitli çalışmalara göre nano-RMCIS'lar daha az flor salmaktadırlar. ${ }^{35,68,69}$ Nano-RMCIS'lardan 
flor salınımında ph 4'de hafif bir artış olmasına rağmen, 84 günün sonunda salınan toplam flor ve gün içinde numune yüzeyi başına salınan flor miktarının geleneksel RMGIC'lerinkiyle karşılaştııılabilir olduğu gözlenmiştir. ${ }^{35}$

Şimdiye kadar nano-iyonomer simanla restore edilmiş dişlerdeki sekonder çürüklerle ilgili uzun dönemli klinik çalışma yapılmamıştır. Ayrıca klinik ortamlarda bu simanların konvansiyonel CIS'lardan daha yüksek çürük önleyici etkisinin olup olmadığı tespit edilememiştir.

\section{SONUÇ VE ÖNERILER}

Konvansiyonel CIS'ların ve RMCIS'ların diş hekimliğinde geniş bir uygulama yelpazesi olmasına rağmen mekanik ve fiziksel özelliklerinin yanısıra flor salınımının arttırıması amacıyla yeni modifikasyonlara intiyaç duyulmuştur. $\mathrm{Bu}$ materyallerin nanomodifikasyonu; RMCIS'lara nano-boyutlu doldurucuların eklenmesiyle, cam partikül boyutlarının azaltıması ve nano boyutlu biyoseramiklerin cam tozuna katıması ile elde edilebilmektedir. Ticari olarak temin edilebilen nano dolduruculu RMCIS'lar, eğilme ve çekme kuvveti açısından mikro dolduruculu RMCIS'lar üzerine anlamlı bir üstünlüğe sahip olmazken, nano dolduruculu RMCIS'ların yapıştırıma özellikleri ve cam-biyoseramik arayüzeyindeki başarısızlık hala endişe konusudur. Dahası, çok az sayıda çalışma nanomodifikasyon CIS'ların pulpa hücreleri üzerine etkilerine odaklanmıştır. Bu nedenle nano-dolduruculu CIS'ların uygulamalarıyla ilgili daha fazla klinik ve laboratuvar çalışmalarına intiyaç olduğu düşüncesindeyiz. 


\section{KAYNAKLAR}

1. Wilson $A D$, Kent BE. A new translucent cement for dentistry: The glass ionomer cement. Br Dent J 1972; 132: 1335.

2. McLean JW. The clinical use of glass ionomer cements. Dental Clinic of North America 1992; 36: 693-711.

3. Sennou HE, Lebugle AA, Gregoire GL. X-ray photoelectron spectroscopy study of the dentin-glass ionomer cement interface. Dent Mater 1999; 15: 22937

4. Preston AJ, Mair LH, Agalamanyi EA, Higham SM. Fluoride release from aesthetic dental materials. J. Oral Rehabil 1999; 26: 123-9.

5. Wiegand A, Buchalla W, Attin T. Review on fluoridereleasing restorative materials-Fluoride release and uptake characteristics, antibacterial activity and influence on caries formation. Dent Mater 2007; 23: 343-62.

6. McLean JW, Powis DR, Prosser HJ, Wilson AD. The use of glass-ionomer cements in bonding composite resins to dentine. Br Dent J 1985; 158:410-4.

7. Abdalla Al, Alhadainy HA, Garcia-Godoy F. Clinical evaluation of glass ionomers and compomers in class V carious lesions. Am J Dent 1997; 10: 18-20.

8. Terata R, Nakashima K, Kubota M. Effect of temporary materials on bond strength of resinmodified glass-ionomer luting cements to teeth. Am J Dent 2000; 13: 209-11.

9. Murdoch-Kinch CA, McLean ME. Minimally invasive dentistry. J Am Dent Assoc 2003; 134: 87-95.

10.De Amorim RG, Leal SC, Frencken JE. Survival of atraumatic restorative treatment (ART) sealants and restorations: A meta-analysis. Clin Oral Investig 2012; 16: 429-41.

11.Peumans $M$, de Munck J, Mine A, van Meerbeek B. Clinical effectiveness of contemporary adhesives for the restoration of non-carious cervical lesions. A systematic review Dent Mater 2014; 30: 1089-103.

12.McCabe JF. Resin-modified glass-ionomers. Biomaterials 1998; 19: 521-7.

13. Hannig $M$, Hannig C. Nanomaterials in preventive dentistry. Nat. Nanotechnol 2010; 5: 565-9.

14. Khurshid Z, Zafar M, Qasim S, Shahab S, Naseem M, AbuReqaiba A. Advances in nanotechnology for restorative dentistry. Materials 2015; 8: 717-31.

15. Najeeb S, Khurshid Z, Matinlinna JP, Siddiqui F, Nassani MZ, Baroudi K. Nanomodified peek dental implants: Bioactive composites and surface modification-A review. Int J Dent 2015; 381759.

16. Le Guéhennec L, Soueidan A, Layrolle P, Amouriq Y. Surface treatments of titanium dental implants for rapid osseointegration. Dent Mater 2007; 23: 844-54.

17. Hannig M, Hannig C. Nanotechnology and its role in caries therapy. Adv Dent Res 2012; 24: 53-7.
18. Terry DA. Direct applications of a nanocomposite resin system: Part 1-the evolution of contemporary composite materials. Pract Proced Aesthet Dent 2004; 16: 417-32.

19. Curtis AR, Palin WM, Fleming GJP, Shortall ACC, Marquis PM. The mechanical properties of nanofilled resin-based composites: The impact of dry and wet cyclic pre-loading on biaxial flexure strength. Dent. Mater 2009; 25: $188-97$.

20.Chen $\mathrm{MH}$. Update on dental nanocomposites. J Dent Res 2010; 89: 549-60.

21. Moshaverinia A, Ansari S, Moshaverinia M, Roohpour N, Darr JA, Rehman I. Effects of incorporation of hydroxyapatite and fluoroapatite nanobioceramics into conventional glass ionomer cements (GIC). Acta Biomater 2008; 4: 432-40.

22. Moshaverinia A, Roohpour N, Chee WWL, Schricker SR. A review of powder modifications in conventional glass-ionomer dental cements. J Mater Chem 2011; 21: 1319-28.

23. Shariq N, Zohaib K, Muhammad SZ, Abdul SK, Sana Z, Juan Manuel NM et al. Modifications in Glass lonomer Cements: Nano-Sized Fillers and Bioactive Nanoceramics. Int J Mol Sci 2016; 17: 1134.

24.De Caluwe T, Vercruysse CW, Fraeyman $\mathrm{S}$, Verbeeck RM. The influence of particle size and flüorine content of aluminosilicate glass on the glass ionomer cement properties. Dent. Mater 2014; 30: 1029-38.

25. Moshaverinia A, Ansari S, Movasaghi Z, Billington RW, Darr JA, Rehman IU. Modification of conventional glass-ionomer cements with $\mathrm{N}$ vinylpyrrolidone containing polyacids, nanohydroxy and fluoroapatite to improve mechanical properties. Dent Mater 2008; 24: 1381-90.

26. Ong JL, Chan DCN. Hydroxyapatite and their use as coatings in dental implants: A review. Crit Rev Biomed Eng 2000; 28: 667-707

27. Huang SB, Gao SS, Yu HY. Effect of nanohydroxyapatite concentration on remineralization of initial enamel lesion in vitro. Biomed. Mater 2009; 4: 034104.

28. Huang S, Gao S, Cheng L, Yu H. Remineralization potential of nanohydroxyapatite on initial enamel lesions: An in vitro study. Caries Res 2011; 45: 460-8.

29. Javed F, Vohra F, Zafar S, Almas K Significance of osteogenic surface coatings on implants to enhance osseointegration under osteoporotic-like conditions. Implant Dent, 2014; 23: 679-86. 
30. Yap AUJ, Pek YS, Kumar RA, Cheang P, Khor KA. Experimental studies on a new bioactive material: Haionomer cements. Biomaterials 2002; 23: 95562.

31.Zakir M, Al Kheraif AAA; Asif M, Wong FSL, Rehman IUA. Comparison of the mechanical properties of a modified silorane based dental composite with those of commercially available composite material. Dent Mater 2013; 29: 53-9.

32.Lucas ME, Arita K, Nishino M.Toughness, bonding and fluoride-release properties of hydroxyapatiteadded glass ionomer cement. Biomaterials 2003; 24: 3787-94.

33. Lee JJ, Lee YK, Choi BJ, Lee JH, Choi HJ, Son HK, Hwang JW, Kim SO. Physical properties of resinreinforced glass ionomer cement modified with micro and nano-hydroxyapatite. J. Nanosci. Nanotechnol 2010; 10: 5270-6.

34.Gu YW, Yap AU, Cheang P, Kumar R. Spheroidization of glass powders for glass ionomer cements. Biomaterials 2004; 25: 4029-35.

35. Moreau JL, $\mathrm{Xu} \mathrm{HH}$. Fluoride releasing restorative materials: Effects of $\mathrm{pH}$ on mechanical properties and ion release. Dent Mater 2010; 26: 227-35.

36. Elsaka SE, Hamouda IM, Swain MV. Titanium dioxide nanoparticles addition to a conventional glass-ionomer restorative: Influence on physical and antibacterial properties. J. Dent 2011; 39: 58998.

37.Garcia-Contreras R, Scougall-Vilchis RJ, ContrerasBulnes R, Sakagami $H$, Morales-Luckie RA, Nakajima H. Mechanical, antibacterial and bond strength properties of nano-titanium-enriched glass ionomer cement. J Appl Oral Sci 2015; 23: 321-8.

38. Garcia-Contreras R, Scougall-Vilchis RJ, ContrerasBulnes R, Kanda Y, Nakajima $H$, Sakagami $H$. Effects of $\mathrm{tio}_{2}$ nano glass ionomer cements against normal and cancer oral cells. In Vivo 2014; 28: 895907.

39.Cattani-Lorente MA, Dupuis V, Payan J, Moya F, Meyer JM. Effect of water on the physical properties of resin-modified glass ionomer cements. Dent Mater 1999; 15: 71-8.

40.Anusavice KJ, Zhang NZ, Shen C. Effect of $\mathrm{CaF}_{2}$ content on rate of fluoride release from filled resins. J Dent Res 2005; 84 440-4.

41.Xie D, Brantley WA, Culbertson BM, Wang G. Mechanical properties and microstructures of glassionomer cements. Dent Mater 2000; 16: 129-38.

42. Mathis RS, Ferracane JL. Properties of a glassionomer/resin-composite hybrid material. Dent Mater 1989; 5: 355-8.

43.Pameijer CH, Garcia-Godoy F, Morrow BR, Jefferies $\mathrm{SR}$. Flexural strength and flexural fatigue properties of resin-modified glass ionomers. J Clin Dent 2015;26: 23-7.

44. Hefferren JJ, Koehler HM. Foods, Nutrition \& Dental Health; Pathotox Publishers: Park Forest South, IL, USA. 1981.
45.Lin J, Zhu J, Gu X, Wen W, Li Q, Fischer-Brandies $H$, Wang $H$, Mehl C. Effects of incorporation of nano-fluorapatite or nano-fluorohydroxyapatite on a resin-modified glass ionomer cement. Acta Biomater 2001; 7: 1346-53.

46. Moraes RR, Gonçalves LdS, Lancellotti AC, Consani S, Correr-Sobrinho L, Sinhoreti MA. Nanohybrid resin composites: Nanofiller loaded materials or traditional microhybrid resins? Oper Dent 2009; 34: 551-7.

47. Mitra SB, 1991. Adhesion to dentin and physical properties of a light-cured glass-ionomer liner/base. J Dent Res, 70: 72-4.

48. Lin A, Mclntyre NS, Davidson RD. Studies on the adhesion of glass-ionomer cements to dentin. J Dent Res 1991; 71: 1836-41.

49. Coutinho E, Cardoso MV, de Munck J, Neves AA, van Landuyt $K L$, Poitevin $A$, et al. Bonding effectiveness and interfacial characterization of a nano-filled resin-modified glass-ionomer. Dent Mater 2009; 25: 1347-57.

50.Kugel G, Ferrari M. The science of bonding: From first to sixth generation. J Am Dent. Assoc 2000; 131: 20-5.

51. Nakaoki Y, Nikaido T, Pereira PNR, Inokoshi S, Tagami J. Dimensional changes of demineralized dentin treated with hema primers. Dent Mater 2000; 16: 441-6.

52.Wilson AD, Prosser HJ, Powis DM.Mechanism of adhesion of polyelectrolyte cements to hydroxyapatite. J Dent Res 1983; 62: 590-2.

53. Korkmaz Y, Gurgan S, Firat E, Nathanson D. Shear bond strength of three different nanorestorative materials to dentin. Oper. Dent 2010; 35: 50-7.

54.El-Askary F, Nassif M. Bonding nano-filled resinmodified glass ionomer to dentin using different self-etch adhesives. Oper Dent 2011; 36: 413-21.

55.Imbery TA, Namboodiri A, Duncan A, Amos R, Best AM, Moon PC. Evaluating dentin surface treatments for resin-modified glass ionomer restorative materials. Oper Dent 2013; 38: 42938.

56. Hamama HH, Burrow MF, Yiu C. Effect of dentine conditioning on adhesion of resin-modified glass ionomer adhesives. Aust Dent J 2014; 59: 193200.

57.Sidhu SK, Schmalz G. The biocompatibility of glass-ionomer cement materials. A status report for the american journal of dentistry. Am J Dent 2001; 14: 387-96.

58. Sauro S, Watson TF, Thompson I, Toledano M, Nucci C, Banerjee A. Influence of air-abrasion executed with polyacrylic acid-bioglass $45 \mathrm{~S} 5$ on the bonding performance of a resin-modified glass ionomer cement. Eur J Oral Sci 2012; 120: 168-77. 
59.Takahashi M, Nakajima M, Tagami J, Scheffel DLS, Carvalho RM, Mazzoni, A. et al. The importance of size-exclusion characteristics of type $\mathrm{i}$ collagen in bonding to dentin matrices. Acta Biomater 2013; 9, 9522-8.

60.Korkmaz Y, Ozel E, Attar N, Ozge BC. Influence of different conditioning methods on the shear bond strength of novel light-curing nano-ionomer restorative to enamel and dentin. Lasers Med. Sci 2010; 25: 861-6.

61.Perdigao J, Dutra-Correa M, Saraceni SH, Ciaramicoli MT, Kiyan VH. Randomized clinical trial of two resinmodified glass ionomer materials: 1 -Year results. Oper Dent 2012; 37: 591-601

62.El Wakeel AM, Elkassas DW, Yousry MM. Bonding of contemporary glass ionomer cements to different tooth substrates; microshear bond strength and scanning electron microscope study. Eur J Dent 2015; 9: 176-82.

63. Forsten L. Fluoride release and uptake by glassionomers and related materials and its clinical effect. Biomaterials 1998; 9: 503-8.

64.Skrtic D, Antonucci JM, Eanes ED, Eichmiller FC, Schumacher GE. Physicochemical evaluation of bioactive polymeric composites based on hybrid amorphous calcium phosphates. J Biomed Mater Res 2000; 53: 381-91.

65. Glasspoole EA, Erickson RL, Davidson CL. A fluoridereleasing composite for dental applications. Dent Mater 2001; 17: 127-33.

66.Ullah R, Zafar MS, Al-Munawwarah AM, Arabia S. Oral and dental delivery of fluoride: A review. Fluoride 2015; 48: 195-204.

67.Zafar MS, Ahmed N. Therapeutic roles of fluoride released from restorative dental materials. Fluoride 2015; 48: 184-94.

68. Neelakantan P, John S, Anand S, Sureshbabu N, Subbarao $C$. Fluoride release from a new glassionomer cement. Oper Dent 2011; 36: 80-5.

69.Paschoal MAB, Gurgel CV, Rios D, Magalhães AC, Buzalaf MAR, Machado M.A.d.A.M. Fluoride release profile of a nanofilled resin-modified glass ionomer cement. Braz Dent J 2011; 22: 275-9.

\section{Yazışma Adresi:}

Doç. Dr. Mustafa Erhan SARI

Ondokuz Mayıs Üniversitesi

Diş Hekimliği Fakültesi

Pedodonti Anabilim Dalı

Kurupelit, Samsun, Türkiye

Tel : +903623121919-2784

Faks : +90362 4576032

E-mail : dterhansari@hotmail.com 